\title{
ROLES DE LOS CENTROS UNIVERSITARIOS MUNICIPALES (CUM) EN EL DESARROLLO LOCAL DE LOS MUNICIPIOS CUBANOS
}

\author{
Carlos A. Hernández M. \\ Centro Universitario Municipal Camajuaní, Cuba. \\ cahm862@uclv.edu.cu
}

(Tipo de Artículo: Reflexión. Recibido el 04/02/2012. Aprobado el 08/05/2012)

\section{RESUMEN}

Se conceptualiza en el aporte de la Educación Superior al Desarrollo Local en los municipios, cómo se inserta en el proceso, las amenazas a su participación y las oportunidades generadas por su inclusión. Se analiza la situación actual y perspectiva y las Estrategias a seguir por la Educación Superior en los municipios. Se define como se trabaja la seguridad alimentaria en función del Desarrollo Local, la superación de Postgrado en el CUM, la formación y superación de los cuadros, la participación en proyectos pertinentes en energía, medio ambiente, servicios a la población, industrias locales y problemas socio - comunitarios y la formación vocacional y articulación pertinente con la Enseñanza Técnico Profesional. Por último se hace una propuesta de Indicadores y herramientas para medir el impacto de la Gestión del Conocimiento y la Innovación dentro del Programa de Desarrollo Local dividida en 2 grupos: Indicadores de funcionamiento interno del CUM e Indicadores de funcionamiento del CUM hacia el Desarrollo Local del Municipio.

\section{Palabras clave}

Educación superior, desarrollo local, gestión del conocimiento y la innovación, postgrado, indicadores.

\section{ROLES OF MUNICIPAL UNIVERSITARY CENTERS (MUC) IN THE LOCAL DEVELOPMENT OF CUBAN MUNICIPALITIES}

\begin{abstract}
A conceptualization is made on the contribution of Higher Education to Local Development in the municipalities, how it is inserted in the process, the threats to their participation and the opportunities generated by their inclusion. The current situation, the perspective and the Strategies to adopt for Higher Education in the municipalities are also analyzed. It is defined how alimentary security works regarding to Local Development, postgraduate degree in MUC, education and self-improvement of Chiefs, participation in pertinent projects in the fields of energy, environment, community services, local industries, social-community problems and vocational education and pertinent articulation with Professional Technician Teaching. Finally a proposal is made about Indicators and tools to measure the effect of Knowledge and Innovation Management within Local Development Program, it has two parts: Indicators of internal operation of the MUC and Indicators of operation of the MUC regarding Local Development of the Municipality.
\end{abstract}

\section{Keywords}

Higher education, local development, knowledge and innovation management, postgraduate degree, indicators.

\section{ROLES DES CENTRES UNIVERSITAIRES MUNICIPALES (CUM) DANS LE DEVELOPEMENT LOCALE DES COMMUNES CUBAINES}

\section{RÉSUMÉ}

On conceptualise sur l'apport de l'Education Supérieure dans le développement local dans les communes, comment s'insère-elle dans le processus, les menaces à son participation et les opportunités produites par son inclusion. On analyse la situation, perspective et les stratégies par rapport à l'Education Supérieur dans les communes. On définit comment est travaillée la sécurité alimentaire dans fonction du Développement Locales, les diplômes de troisième cycle dans MUC, la formation et dépassement des Chefs, la participation dans projets pertinents dans énergie, environnement, services pour la population, industries locales et des problèmes sociocommunautaires, la formation vocationnelle et l'articulation pertinent avec l'Enseignement Technique Professionnelle. Finalement on propose des indicateurs et outils pour mesurer l'effet de la gestion de la connaissance et l'innovation à l'intérieur du Programme de Développement Locale en deux parties : Indicateurs de fonctionnement interne du CUM et indicateurs de fonctionnement du CUM par rapport au Développement Local de la Commune.

\section{Mots-clés}

Education supérieure, développement locale, gestion de la connaissance et l'innovation, études de troisième cycle, indicateurs. 
"La auténtica dignidad de cualquier ser humano radica en la satisfacción de poseer un trabajo estimulante, de ser capaz de desarrollarse en él y de encontrar que lo que hace es valioso" Shigeru Kboyaschi

\section{INTRODUCCIÓN}

Todos los modelos contemporáneos de relación universidad-sociedad se basan en la necesidad de trabajar en redes. por tanto los CUM, para cumplir su misión, necesitan realizar su trabajo en redes cuyos actores sean el gobierno local, las otras filiales del territorio, las bibliotecas y centros de información, las empresas, los representantes locales de ministerios como el CITMA, las organizaciones políticas, sociales y profesionales y los movimientos sociales como el fórum de ciencia y técnica.

La gestión del conocimiento en este contexto consiste en gran medida en colaborar en la identificación de problemas locales que lo requieran para su solución y en contribuir a identificar las organizaciones 0 personas que pueden aportarlo, para luego construir los nexos, las redes y los flujos de conocimientos que permitan su asimilación, evaluación, procesamiento y uso. Los CUM pueden actuar como agentes relevantes en la construcción social del mismo y en el establecimiento de las conexiones que permitan el flujo entre los actores de la red local de su territorio [1].

La producción de conocimiento a nivel local plantea varios retos de gran complejidad:

1. Frecuentemente, los problemas son complejos y necesitan un abordaje multi o inter-disciplinario, requiriendo de la integración de varias disciplinas en la búsqueda de respuestas cuya solución muchas veces se logra combinando inteligentemente los conocimientos existentes.

2. Generalmente, el conocimiento que se requiere está integrado a la práctica, se necesita para resolver un problema y existe gran parte del mismo, por lo que sólo es necesario transferirlo con creatividad teniendo en cuenta la singularidad de las circunstancias locales.

3. Existe un fuerte nexo entre innovación y aprendizaje por lo que para introducir lo nuevo antes hay que capacitar al personal que trabajará en el proceso.

El primer reto obliga a tener un levantamiento de información de los profesionales del territorio acompañada de los conocimientos que manejan y en qué pueden aportar al enfoque multi-disciplinario de la solución de cualquier problema. El segundo reto crea la necesidad del aprendizaje por parte de los actores locales de la red, para realizar las funciones que les corresponden en el contexto de la misma. Esto lleva a la necesidad de un programa de educación continua de estos profesionales que engrana con el tercer reto, al realizar esa capacitación y superación para prepararlos de cara a enfrentar los nuevos conocimientos generados.
De acuerdo con Lage [2], el "conocimiento relevante" producido a nivel local se debe caracterizar por ser: (a) colectivo, incorporado a las organizaciones, (b) combinatorio, de fuentes y disciplinas diversas, (c) concreto, vinculado a la solución de problemas específicos, (d) tácito, frecuentemente no estructurado y (e) local, lo que ilustra el tipo de conocimientos que deberán gestionar los CUM para atender los problemas del territorio que los rodea.

En la gestión del conocimiento a nivel local se debe eliminar las distancias que se han creado artificialmente entre las disciplinas, entre los actores y entre los procesos de aprendizaje, de superación, de capacitación, de investigación y de innovación. Esto es importante porque obliga a todos los actores a trabajar integradamente en función de la obtención de un conocimiento utilizable. Es imprescindible encaminar acciones para optimizar los recursos humanos disponibles y dar un trato adecuado y justo a las personas que integran la organización, lo que requiere una dirección centralizada de recursos que posibilite proyección prospectiva que favorezca los saltos cualitativos que se deriven de los cuantitativos. Con ello se avanza hacia una integración estratégica [3].

En un CUM, el logro de una dirección eficiente, eficaz e integrada [4] revierte singular importancia porque lleva aparejado una serie de acciones encaminadas sobre las concepciones educativas y curriculares asumidas que declaran el carácter nacional, científico, democrático, popular y político en un proceso de vinculación y mutua influencia con la sociedad. Además, lleva implícito el desarrollo de la investigación científico-tecnológica de dicha institución, lo que repercute en el apoyo intelectual a la teoría de las ciencias y en la economía productiva y social de la población y realiza una interacción integral con la comunidad a través de sus actividades de extensión.

En 2002 se inició un proceso de transformaciones de la educación superior con el objetivo de garantizar el pleno acceso de la población a todas las actividades sustantivas, cultivando su inteligencia y multiplicando gradualmente sus conocimientos. A partir de los recursos materiales y humanos creados durante estos fructíferos años de revolución y que hoy se encuentran diseminados por toda la Isla [5].

Para dar respuesta a estas nuevas exigencias surgen los Centros Universitarios Municipales CUM y se inicia un amplio programa de Universalización en Cuba. La nueva universidad cubana, internacionalista y abierta a toda la sociedad, se distingue de la tradicional que existe en la mayoría de los países del mundo, porque trasciende sus muros y desarrolla sus procesos en íntima comunidad con el pueblo, perfeccionándose continuamente como parte de una interrelación en la que todos deben participar. 
En su primera etapa, los CUM se dedicaron a garantizar la continuidad de estudios con la calidad requerida en las carreras que se imparten y se han incorporado otros procesos sustantivos de la vida universitaria como respuesta a las necesidades del desarrollo de cada territorio. Estas misiones imponen la necesidad de lograr con urgencia la integración y cooperación de todos los factores del territorio para elevar la calidad, racionalidad y pertinencia de los resultados de las instituciones. Esa es la única forma de dar respuesta a las complicadas demandas que plantea el gobierno y las instituciones del territorio. A tono con las ideas anteriores, el punto de partida para identificar el papel y el lugar de la universidad actual y con ello poder establecer de la mejor forma posible su modelo de formación, se debe buscar en la propia misión de la universidad. Según Horruitiner [6], consiste en preservar, desarrollar y promover, a través de sus procesos sustantivos y en estrecho vínculo con la sociedad, la cultura de la humanidad.

La misión asignada a la nueva universidad cubana en las nuevas condiciones de la municipalización conduce a la siguiente interrogante: ¿Cómo lograr la integración de todos los factores del territorio garantizando el desarrollo local y el cumplimiento de la misión de la educación superior bajo las condiciones de la municipalización?

\section{DESARROLLO}

Los CUM deben actuar en el contexto del municipio como gestores de conocimiento y la innovación y, para cumplir esa función, deben minimizar las amenazas y potenciar las oportunidades que se presenten en el entorno municipal a partir de las transformaciones en la educación superior y buscando la calidad, la eficiencia y la racionalidad económica. Además, aportan al desarrollo local identificando los problemas, potenciando la capacitación orientada a las necesidades, conectando el conocimiento a las necesidades sociales, colaborando en la gestión del conocimiento que facilitará las soluciones y formando valores en todos los actores locales.

\subsection{Amenazas a la participación del CUM}

- Insuficiencia de conocimiento, concientización y herramientas metodológicas para la acertada incorporación del conocimiento como recurso imprescindible para el desarrollo local sostenible.

- Limitada integración y coordinación interinstitucional y sectorial en la gestión del conocimiento.

- Inadecuada incorporación de la gestión del conocimiento a los planes y programas del municipio.

- Limitados recursos financieros e incentivos favorables a la gestión del conocimiento a escala de municipio, consejo popular y comunidad.

- Tendencia a la disminución de las matrículas y carreras en los CUM.

- Voluntarismo en la superación de directivos y profesionales del territorio.

- Estilos de trabajo que no propician el cambio.
- Falta de visión de los decisores en los organismos municipales sobre el papel del CUM como institución dinamizadora de la gestión del conocimiento y de la innovación en el territorio.

- Débil formación vocacional que no está orientada a necesidades del territorio.

- Articulación no pertinente de la enseñanza técnico profesional con la educación superior.

- Poca generalización de las investigaciones que se realizan en los planes de ciencia y técnica.

- Debilidad en dimensión jurídica y en ciencias sociales.

- Existencia de empresas no subordinadas localmente.

\subsection{Oportunidades generadas por la participación}

- Difusión de los resultados en los eventos y congresos nacionales e internacionales.

- Posibilidad de capacitar a los diferentes actores en función de las prioridades del territorio.

- El Pilotaje del MES a la IMDL posibilita el nexo sede Central-CUM-Gobierno en el municipio.

- La articulación entre los actores locales y el nexo entre el saber académico y el saber popular viabiliza aplicar la máxima de pensamiento global y actuación local.

- Presencia de una red de actores del territorio y su articulación con otras redes.

- Papel protagónico del gobierno en el desarrollo local.

- Integración pertinente de las funciones sustantivas de la educación superior para el desarrollo local.

- Integración de todos los organismos formadores en función del desarrollo local.

\subsection{Retos de la gestión del conocimiento y de la innovación}

- Perfeccionar labor educativa de profesores y estudiantes.

- Coadyuvar a un desarrollo humano sostenible.

- Lograr la integración entre la red de actores.

- Promover una investigación científica y pertinente.

- Lograr la Pertinencia universitaria.

- Formación de pregrado y postgrado orientada al desarrollo local.

- Usar recursos financieros a partir de proyectos.

- Uso acelerado de las tecnologías.

- Articular las acciones de iniciativas con programas, redes, organizaciones e instituciones locales, con el foco del desarrollo municipal y seguridad alimentaria.

- Lograr un desarrollo social y económico sostenible con base en el conocimiento.

\section{SITUACIÓN ACTUAL Y PERSPECTIVA DE LA EDUCACIÓN SUPERIOR EN LOS MUNICIPIOS}

Hasta hoy el desarrollo local estaba implícito en el trabajo de la educación superior hacia el municipio, pero no era priorizado frente a la formación de profesionales. Hoy el desarrollo local pasa a ser una prioridad, pero la formación profesional en los CUM disminuye con tendencia a desaparecer, lo que hace 
que la pertinencia pase a ser casi virtual. Perder la integración pertinente de las funciones sustantivas con el desarrollo del territorio debido a la disminución acelerada de la formación profesional en el CUM, afecta el desarrollo Local. Se hace necesario, en esas complejas condiciones, un cambio de paradigma para los CUM debido a la necesidad de la universalización del conocimiento con equidad y justicia social, de lograr calidad y pertinencia con respuesta a las demandas del desarrollo local.

Surge entonces este interrogante [7]: ¿Cómo aprovechar la oportunidad que brinda la prioridad recibida por el desarrollo local, conjurando la amenaza de la disminución de la formación profesional en los CUM, potenciando algunas fortalezas y neutralizando algunas debilidades existentes, para potenciar el desarrollo local con visión desde el CUM?

\subsection{Estrategias de la educación superior en los municipios}

Ante esa combinación de factores, que pueden potenciar o afectar el desarrollo local, es necesario desarrollar estrategias para aprovechar las oportunidades externas que ofrecen las prioridades para el desarrollo local y que han dado organismos para posibilitar el acceso a los créditos para los proyectos, la creación de las Delegaciones Municipales del MINAG, la atención de la comisiones de la ANPP al desarrollo local y las prioridades otorgadas por el CITMA a las ciencias sociales y humanísticas.

Otra estrategia es potenciar las fortalezas logradas en el sistema MES para el desarrollo local, expresadas en el Lineamiento $10 / 2010$ del MES, la experiencia acumulada en el Programa Ramal de Ciencia y Tecnología GUCID, la integración de las funciones sustantivas de la educación superior, la experiencia en la capacitación de actores y formación de capacidades a nivel de municipio. También incluyen a la Integración de sedes centrales y los CUM, la capacitación y educación de postgrado para la innovación realizada en el entorno local y las experiencias sistematizadas por algunos doctorados que se realizan en temas de desarrollo local.

Como estrategia para neutralizar las debilidades en función del desarrollo local se debe lograr mayor articulación de las redes locales del MES y otras que operan en los municipios, fortaleciendo la capacidad de accionar del GUCID en los CUM. Se debe elevar la pertinencia e integración de las funciones sustantivas para el desarrollo local, aprovechar las posibilidades que brindan los proyectos de la IMDL y el MEP y participar en otros pilotajes y proyectos municipales en energía, medio ambiente, industria y servicios. Se debe potenciar la integración pertinente entre las sedes centrales y los CUM, el uso de estudiantes del municipio que estudian en las sedes en proyectos de producción de alimentos, vivienda o TIC y desarrollar y aplicar un sistema de indicadores GUCID para el desarrollo local.
La estrategia para conjurar la amenazas que acechan al desarrollo local debe aprovechar las posibilidades que brinda la función estatal de asesoría a la proyección estratégica del CAM, determinar las demandas reales de formación profesional a partir de los problemas y oportunidades existentes en el territorio y realizar una articulación consecuente de la agenda formativa entre la educación superior, la enseñanza media y la enseñanza técnico profesional. También se debe realizar una formación vocacional consecuente para el desarrollo local, una preparación dirigida al ingreso a la educación superior en función del desarrollo local, una gestión efectiva de matrícula en las carreras del CUM determinantes para el desarrollo local y un manejo adecuado del plan de estudios propio y electivo en función del mismo.

Si se aprovechan las oportunidades de prioridad del desarrollo local, se conjuran las amenazas, se aprovechan las fortalezas y se neutralizan las debilidades mediante las capacidades creadas por el programa ramal GUCID en los CUM, es posible salvar el núcleo duro de perfiles de carreras y los CUM imprescindibles para realizar avances importantes en el desarrollo local.

\subsection{Seguridad alimentaria en función del desarrollo local}

La seguridad alimentaría esta íntimamente ligada al desarrollo local, sobre todo a partir de que la mayor parte de los municipios del país tienen una economía con un fuerte carácter agropecuario. La elaboración de la estrategia para la agricultura suburbana en un radio de $5 \mathrm{~km}$. del municipio y 3 de cada consejo popular es un proceso en que los CUM pueden aportar [8].

La industria transformadora y conservadora de alimentos, que se deberá desarrollar en el país, jugará en muchos municipios un papel clave en el proceso de su industrialización mediante el desarrollo de la pequeña y mediana empresa. Para esto se requieren varias soluciones de financiamiento, energía y recursos humanos, es necesario encontrar financiamiento en los planes de la economía, sistema crediticio nacional que aún está por desarrollarse, los proyectos de IMDL del MEP, proyectos internacionales, sobre todo a través del ALBA, donativos y otras fuentes.

\subsection{Papel del CUM en la formación y superación de los cuadros para las necesidades del desarrollo local}

A partir de la indicación del MES en 2005, las sedes universitarias municipales se convirtieron en figura protagónica en la preparación y superación de los cuadros y reservas del territorio. Para acometer esta tarea se usan todas las figuras de superación del postgrado y diferentes alternativas que incluyen la semi-presencialidad y capacitación desde el puesto de trabajo, aunque durante algunos años predominaron los cursos.

Se crearon los grupos de trabajo municipales para la formación y superación de los cuadros y reservas, 
integrados por el profesor de la CUM que atiende la actividad, el funcionario que atiende cuadros en la AMPP, un grupo de entrenadores de cuadros de mayor experiencia y los capacitadotes de los OACE y otros organismos. Su función es asesorar y controlar el proceso de planeación estratégica e introducción de otras técnicas avanzadas de dirección y en las actividades de preparación y superación de los cuadros y reservas.

El proceso de conciliación de la estrategia de preparación y superación de los cuadros y reservas se ha sistematizado. Las conciliaciones de la estrategia de preparación y superación de los cuadros en el CAM, con participación de la CUM, llegan hasta las empresas y organismos de base como parte de un sistema de trabajo y avanzando en la implementación de la estrategia maestra de preparación política e Ideológica. Es una fortaleza el seguimiento dado por las sede centrales de la universidad a la formación académica de los cuadros y reservas. Ellos se han insertado en los diferentes programas de maestrías en dirección y otros están en formación, dentro de los cuales se encuentran directivos de empresas y organismos, presidentes de consejos populares, presidentes, vicepresidentes y secretarios de CAM. También hay cuadros egresados y matriculados en maestrías de otras ramas del saber afines con la labor que realizan, como la maestría de energía y la maestría de educación superior para los que imparten docencia en el CUM.

\subsection{Formación de profesionales en el CUM en función de las necesidades del desarrollo local}

La necesidad de formar a los profesionales en las instituciones de la educación superior del municipio en las especialidades necesarias para el desarrollo local es una prioridad. Por tanto las propuestas de necesidades de matrícula deben realizarse con una planificación a corto, mediano y largo plazo que responda al plan de desarrollo municipal que deriva del diagnóstico de necesidades del territorio.

Por lo general, esos estudios dan como resultado que en los municipios escaseen los profesionales especializados en la producción de alimentos, el desarrollo industrial, la planificación y control de la economía, el trabajo con los recursos humanos y las ciencias sociales. Esto indica la necesidad de mantener un núcleo fuerte en las carreras agropecuarias, industrial, contabilidad y finanzas y quizás alguna presencia de sociología o estudios socioculturales. Para ello es necesario priorizar estas carreras en los planes de matrícula y promover la formación profesional, sobre todo en las menos atractivas.

\subsection{Superación de postgrado en el CUM en función de las necesidades del desarrollo local}

Para desarrollar las acciones de postgrado se ha diseñado un plan anual de superación postgraduada a nivel de sede central, las facultades y los CUM a partir del diagnóstico de las necesidades de los principales organismos e instituciones del territorio. Para lograr una mayor satisfacción de las necesidades determinadas, se ha trabajado de forma integrada con la red del MES. Se parte de las demandas de los profesionales en lugar de hacer una simple oferta de cursos y otras formas de superación; se incluyen en esta modalidad formas de capacitación que no van dirigidas directamente a profesionales pero que resuelven necesidades locales de formación en trabajos de técnicos, obreros y oficios manuales.

En la superación postgraduada se ha desarrollado diversos cursos, entrenamientos, diplomados y actividades dinámicas y en el postgrado académico, maestrías, especialidades y doctorados. Las principales temáticas a abordar en la actividad postgraduada para el desarrollo local giran alrededor de temas de manejo de recursos hídricos, biofertilizantes, abonos orgánicos, manejo integrado de plagas, lombricultura, salud y nutrición animal, manejo y conservación de suelos, producción de viandas y granos, educación ambiental, economía y desarrollo local, gestión de proyectos, uso de sistemas de información geográfica, proyección para la producción agropecuaria sostenible en pequeñas fincas, producción de alimentos en ecosistemas frágiles, idiomas, informática, pedagogía, ciencias sociales, economía y contabilidad, salud y energía entre otros.

Las lecciones aprendidas en el postgrado indican que no siempre los DNA son lo suficientemente objetivos para diseñar la Superación, porque no aplican los instrumentos apropiados para realizarlas, hay limitada utilización de enfoques y métodos de avanzada en la formación de postgrado, existen insuficientes recursos logísticos y bibliográficos físicos y digitalizados para desarrollar las actividades presenciales y semipresenciales. También son restringidos los espacios para la comunicación, difusión y retroalimentación de las informaciones de los programas socioeconómicos, en los cuales participen autoridades de los órganos y organismos.

Los resultados a esperar del postgrado se concentran en nuevos y mejorados productos, servicios, procesos y tecnologías, mayor satisfacción de la población, incrementos de calidad de vida, disminución de pérdidas y afectaciones a la economía nacional e individual e incremento de efectividad en el modo actuar.

\subsection{Participación en proyectos pertinentes en energía, medio ambiente, servicios, industrias locales y problemas socio-comunitarios}

Se debe capacitar a los actores locales en gestión energética empresarial para elevar las capacidades técnico-organizativas de las empresas para la mejora continúa de su eficiencia energética. Hay necesidad de realizar una fuerte gestión ambiental en colaboración con el CITMA y todos los organismos del territorio; se debe potenciar la aplicación de un sistema de acciones socio-culturales e incremento del nivel de participación comunitaria por la introducción de la metodología y estrategia para el trabajo comunitario en los consejos populares. 
Se deben realizar estudios en el municipio para incluir proyectos en la IMDL. Priorizar proyectos sobre producción de materiales para la construcción, financiado por PDHL, hábitat y otras iniciativas. A través de la vinculación de las carreras de ciencias sociales y humanísticas se realizarán en el territorio diferentes intervenciones sobre el diagnóstico y la caracterización de problemas del hábitat y la convivencia en asentamientos humanos. Hay que lograr la integración de las redes de actores municipales y su articulación a otras redes MES y nacionales para el desarrollo local participando en el movimiento de vaquerías escuelas y producción de alimento animal del ICA y en la entrega de tierras en cada municipio.

Se mantendrán las páginas web de los CUM con visibilidad en la intranet del MES, perfeccionando el proyecto de informatización del gobierno y un grupo de organismos clave en el municipio, como el CUM, MINED, salud pública, la oficina municipal de estadística, vivienda y varias entidades de la agricultura. Se debe diseñar el portal municipal con una concepción descentralizada. Se necesita un diagnóstico de los recursos informáticos materiales y humanos disponibles y la conectividad tanto en el gobierno como en las entidades de subordinación local y nacional de mayor interés para a partir del proyecto de Informatización de la Sociedad.

La participación en el proyecto de innovación agrícola local de extensión al municipio de la bio-diversidad y los piensos locales permite la realización de ferias de productores en el municipio, entrega de semillas a los productores, capacitación en tecnologías apropiadas y socialización de Procesos de Innovación para el desarrollo Municipal. Es una iniciativa que se debe aprovechar en función de la producción sostenible de alimentos en la localidad.

\subsection{Formación vocacional y articulación pertinente de enseñanza técnico profesional con educación superior para el desarrollo local}

Con la participación de todos los actores locales implicados se debe lograr:

- Integración con el grupo de formación vocacional del Ministerio de Educación.

- Vinculación con los politécnicos agropecuarios en la selección de estudiantes que aseguren las carreras agropecuarias para su preparación al ingreso en el CUM.

- Orientar la formación técnico profesional y la capacitación a los objetivos del desarrollo local del municipio, a través de propuestas curriculares integradoras de nivel superior y medio, orientadas al desarrollo local y propuestas curriculares de capacitación orientadas a oficios para el desarrollo local.

- Gestión efectiva para matrícula de carreras determinantes para el desarrollo local.

\subsection{Propuesta de indicadores y herramientas para medir el impacto de la gestión del conocimiento y la innovación dentro del programa de desarrollo local}

Tras comparar las propuestas de un gran grupo de municipios participantes en los proyectos del programa ramal GUCID, a continuación se enumera un grupo de indicadores en que se logró consenso para considerarlos herramientas para evaluar el impacto de la labor del CUM en función del desarrollo local.

Grupo 1: Indicadores de funcionamiento interno del CUM:

- Mejora de la estructura de categorías docentes de los claustros.

- Plan de superación personalizado de todos los trabajadores del CUM.

- Mejora en indicadores de control al PDE e indicadores docentes en pregrado y postgrado.

- Está actualizada la demanda de profesionales y de superación por perfil.

- Participación en la planeación estratégica del consejo de la administración municipal.

- Capacitación directa a productores, cuadros y profesionales en todas las formas productivas.

- Creación del grupo de desarrollo local sede centralCUM-Gobierno-CITMA-PDHL.

- Renovadas las líneas de investigación de las carreras para el DESARROLLO LOCAL.

- Impacto en la producción de alimentos por el acompañamiento de proyectos financiados por el IMDL.

- Integradas y coordinadas las acciones de gestión del conocimiento e innovación entre instituciones y sectores que tributan al programa de la IMDL.

- Se implementa el sistema de información gerencial y de informatización.

Grupo 2: Indicadores de funcionamiento del CUM hacia el desarrollo local municipal:

- Uso de sistemas información geográfica para planificación local y entrega de tierras.

- Fortalecimiento de la gestión económica de empresas y organismos del territorio.

- Impacto sobre los volúmenes y la eficiencia de la producción.

- Impactos sobre el desarrollo Infraestructural.

- Impactos sobre el empleo y la productividad del trabajo.

- Impactos medioambientales.

- Impactos sobre el nivel de vida de la población y de grupos vulnerables.

- Impactos sobre el desarrollo científico tecnológico y del capital humano.

- Nuevas prácticas de manejo del recurso agua introducidas por los productores.

- Se realizan acciones de conservación y mejoramiento de suelo en las áreas seleccionadas.

- Identificadas las áreas y aplicados los paquetes tecnológicos apropiadas para las producciones de arroz, frijol y humus de lombriz en los consejos populares, empresas y cooperativas seleccionadas. 
- Incremento de las áreas boscosas del municipio. Recuperación de la cuenca de los ríos.

- Disminución de los índices de degradación de los suelos por la aplicación de tecnologías de preparación de suelos conservacionistas, manejo adecuado y la mayor cobertura de los suelos.

- Mejoramiento y conservación de la diversidad biológica de cultivos de importancia económica a través de la introducción de nueva variedades de fríjol, maíz, boniato, yuca, tomate y soya.

- Reducción del nivel de delincuencia y la reinserción del $100 \%$ de los ex reclusos en las comunidades.

\section{CONCLUSIONES}

- La educación superior debe actuar en el municipio como gestor de conocimiento e innovación.

- Se debe minimizar las amenazas y potenciar las oportunidades que se presenten en el municipio para cumplir su función, a partir de las transformaciones en la educación superior con calidad, eficiencia y racionalidad.

- Perder la integración pertinente de las funciones sustantivas con el desarrollo del territorio debido a la disminución acelerada de la formación profesional en el CUM, afecta el desarrollo local.

\section{AGRADECIMIENTOS}

El autor reconoce las contribuciones de Israel Alonso Díaz, Bárbaro Cala Cristián y Mabel Claro González a la versión original de este documento. Agradece también a los organizadores del programa ramal GUCID del Ministerio de Educación Superior Dr. Jorge Nuñez Jover,
Dra. Aurora Fernández, MSc. José Lázaro Hernández, Lic. Roberto Garcés y Dr. José Luís García Cuevas por la formación lograda con su apoyo dentro de GUCID en la fijación de los criterios teóricos sobre este tema.

\section{REFERENCIAS}

[1] J. Núñez; L. Montalvo \& I. Pérez. "La gestión del Conocimiento, la Ciencia, la Tecnología y la Innovación en la Nueva Universidad: una aproximación conceptual". Revista Pedagogía Universitaria, Vol. XI, No. 2, pp. 114, 2006.

[2] A. Lage. Intervención en el Taller Nacional sobre Gestión del Conocimiento en la Nueva Universidad. 27 Junio - 1 de Julio. La Habana, Cuba. 2005.

[3] K. Cloke \& J. Goldsmith. "El fin del management y el surgimiento de la democracia organizacional: Guía práctica para el puesto de trabajo del futuro". John Wiley \& Sons, 2002.

[4] P. G. Ronda. "Modelo de dirección estratégica para organizaciones de seguridad y protección en el contexto económico cubano". PhD. Tesis, CETDIR, La Habana, Cuba. 2002.

[5] Ministerio de Educación Superior. "La Universidad que queremos". Oficina del Viceministro Primero, La Habana, Cuba. 2004.

[6] P. Horruitinier. "La Universidad Cubana: el modelo de formación”. Ed. Félix Varela, 2008.

[7] J. L. García. "CUM: Gestores de Conocimiento y la Innovación. Amenazas y Oportunidades a partir de la Calidad y Pertinencia". Primer Taller de Segunda Etapa del PR GUCID, 2010.

[8] A. Rubio. "Sobre la seguridad alimentaria". Primer Taller de Segunda Etapa del PR GUCID, 2010. 